\title{
Chlorophyll Segmentation Of Satellite Image With Region-Based Active Contours
}

\author{
Margaretha Rozady ${ }^{1}$ \\ ${ }^{1}$ Informatics Engineering Department, Atma Jaya University \\ Yogyakarta \\ E-mail : margareth_85@yahoo.com \\ Pranowo $^{3}$ \\ ${ }^{3}$ Informatics Engineering Department, Atma Jaya University \\ E-mail : pran@mail.uajy.ac.id
}

\begin{abstract}
In image processing, segmentation is a complex task that requires the use of an accurate method. Interpretation of satellite image today is important in remote sensing tasks. A defined area of satellite image segmentation to be performed. The purpose of this study was to use a method for extracting boundary Active Contours of chlorophyll from satellite imagery, so it can be known areas that contain chlorophyll and areas that do not contain chlorophyll. The results demonstrate the effectiveness of this method of segmentation between the chlorophyll and the surrounding areas that contain little or no chlorophyll.
\end{abstract}

Keyword: chlorophyll, segementation, satellite image, active contour, level set methods.

\section{INTRODUCTION}

Today satellite remote sensing systems provide large volumes of data that are invaluable in monitoring Earth resources and the effects of human activities. Road feature extraction from remotely sensed images has been a longterm topic of research and because of its complexity is still a challenging topic[7]. The development and application of various remote sensing platforms result in the production of huge amounts of satellite image data [2].

Segmentation is a key step before performing higher level operations such as recognition,scene understanding, indexing etc. Applications range from areas related to agriculture, environment study, geophysical exploration to even national security and military related tasks. In all these domains, the quality of the final result depends largely on the quality of the segmentation of the satellite images[9].

Phytoplankton is a major autotroph organisms in marine life. With the process of photosynthesis, phytoplankton can be a source of energy for the entire marines through the food chain. One contained in plankton pigments are chlorophyll that are considered optically active components. Approximately 1 to $2 \%$ of the dry weight of planktonic algae, it has been considered an indicator of algal biomass and thus the trophic state of aquatic environments [3].

Active contour methods have become very popular in recent years, and have found applications in a wide range of problems including visual tracking and image segmentation [9]. The

\author{
Herman Kabetta ${ }^{2}$ \\ ${ }^{2}$ Informatics Engineering Department, Atma Jaya University \\ Yogyakarta \\ E-mail : betabox@rocketmail.com
}

basic idea is to allow a contour to deform so as to minimize a given energy functional in order to produce the desired segmentation. Two main categories exist for active contours: edge based and region-based [4][10] . Edge-based active contour models typically use image gradient as an image-based force to attract the contour toward object boundaries [11] whilst region-based methods look for uniformity within an image sub-region, based on some consistent property such as intensity,colour or texture [6] [5] [1].

Image segmentation is a fundamental problem in image processing and computer vision. Extensive study has been made and many techniques have been proposed, among which the Active Contours is one of the most successful methods. The basic idea of active contours is to evolve a curve under some constraints to extract the desired object [8].

This paper evaluate the effectiveness of using this method for chlorophyll segmentation.

\section{ACTIVE CONTOUR}

Active contour, also called as snake was first proposed by Kass in 1988, its a spline that uses the energy minimization procedure to impose a contour toward a particular feature. Active contour is a popular method in computer vision as well as digital image processing area. Examples of the usefulness of active contour is the observation of the organ in the fields of medicine [1], identification of characters in the postal letter. Snake is mathematically represented using the following equation:

$$
E_{\text {snakg }}=\int_{s=0}^{1} E_{\text {int }}(v(s))+E_{\text {img }}(v(s))+E_{c o n}(v(s)) d s(1)
$$

where:

- $\mathrm{E}_{\text {snake }}$ is the energy function of the active contour from which an object is detected.

- $v(s)$ is the contour (curve).

- Eint is the internal energy of the active contour affecting the motion of the contour.

- $\mathrm{E}_{\mathrm{img}}$ is energy from the input image including low level features such as edge points.

- $\mathrm{E}_{\mathrm{s}}$ is the external constraint imposed by either users or high level processes. 
Eint is given by :

$$
E_{\text {int }}=\alpha(s)\left|\frac{d v(s)}{d g}\right|^{2}+\beta(s)\left|\frac{d^{2} s(s)}{d g^{2}}\right|^{2}
$$

where:

- The derivative $d v(d) / d s$ calculates the energy affecting the elasticity of the contour.

- $\alpha(s)$ is the parameter for elasticity.

- The second derivative $d^{2} v(d) / d s^{2}$

calculates the energy affecting the curvature of the contour

- $\beta(s)$ is the parameter for curvature.

The active contour formula shown in Equation 1 is further expressed numerically using the following equation :

$$
A_{x}=f_{x}(x, y)
$$

where $f x(x, y)$ is the edge magnitude of the image, and $\mathrm{A}$ is a penta-diagonal matrix having the following pattern:

$$
A=\left[\begin{array}{ccccccc}
c_{1} & d_{1} & e_{1} & 0 & \ldots & a_{1} & b_{1} \\
b_{2} & c_{2} & d_{2} & e_{2} & 0 & \ldots & a_{2} \\
a_{3} & b_{3} & c_{3} & d_{3} & e_{3} & 0 & \ldots \\
0 & \ldots & \ldots & \ldots & \ldots & \ldots & \ldots \\
\ldots & \ldots & \ldots & \ldots & \ldots & \ldots & \ldots \\
e_{s-1} & 0 & \ldots & a_{s-1} & b_{s-1} & c_{s-1} & d_{s-1} \\
d_{s} & e_{s} & 0 & \ldots & a_{s} & b_{s} & c_{s}
\end{array}\right]
$$

The values of $a, b, c, d$ and $e$ can be obtained by using the following equations:

$$
\begin{aligned}
& \alpha_{g}=\frac{\beta_{s-1}}{\hbar^{4}} b_{g}=-\frac{2\left(\beta_{s}+\beta_{s-1}\right)}{\hbar^{4}}-\frac{\alpha_{s}}{\hbar^{2}} c_{g}=\frac{\beta_{s+1} \beta_{s} \beta_{s-1}}{\hbar^{4}}+\frac{\alpha_{s+1 \pi_{s}}}{\hbar^{2}} d_{g}= \\
& -\frac{2\left(\beta_{x+1}+\beta_{s}\right)}{\hbar^{4}}-\frac{\alpha_{s+1}}{\hbar^{2}} e_{g}=\frac{\beta_{s+1}}{\hbar^{2}} e_{g}=\frac{\beta_{s+1}}{\hbar^{4}}
\end{aligned}
$$

where $s$ represents the index of the contour points and $\mathrm{h}$ is the space between points in the contour. An iterative solution can then be formulated as follows:

$$
x^{(i+1)}=\left(A+\frac{1}{\Delta} I\right)^{-1}\left(\frac{1}{\Delta} x^{(0)}+f_{x}(x, y)\right)
$$

where $I$ is an identity matrix, $x^{i+1}$ is the new $x$ position of the contour, and delta is a step size. The $y$ position of the contour points can be obtained in similar way using the following equation:

$$
y^{(i+1)}=\left(A+\frac{1}{d} I\right)^{-1}\left(\frac{1}{d} y^{(0)}+f_{y}(x, y)\right)
$$

\section{EXPERIMENT RESULT}

To evaluate the effectiveness of active contour methods we apply the method to satellite images that contain chlorophyll. The original image is an image that has the distribution of chlorophyll. The color red indicates the highest levels of chlorophyll.

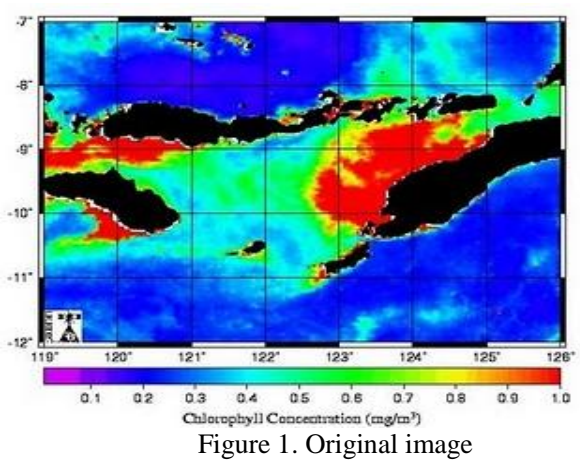

This method has been tested empirically following 5 parameters:

(1) seed

User is asked to provide a seed point from which the contour will expand.

(2) $\sigma$

$\sigma$ is used in Gaussian filter to control the range of influence of the image edges.

(3) $\alpha$ and $\beta$

Two parameters are required in the Sigmoid Image Filter to define the linear transformation to be applied to the sigmoid argument; we call them $\alpha$ and $\beta$.The two parameters are used to intensify the differences between regions of low and high values in the speed image.

(4) Stopping Value

Stopping Value is desirable to stop the process once a certain time has been reached.

The value of the parameters are : $\sigma=0.1, \alpha=0.2, \beta=$ 2 , seed $=(30.50)$, stopping value $=300$. Figure $1-3$ shows the iterations that occur up to a maximum of 300 iterations.

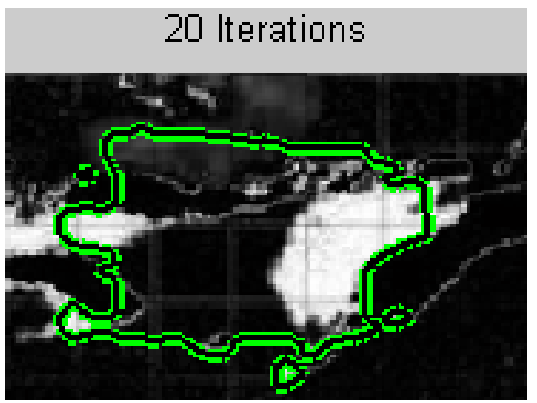

Figure 2. Result of segmentation with 20 iteration

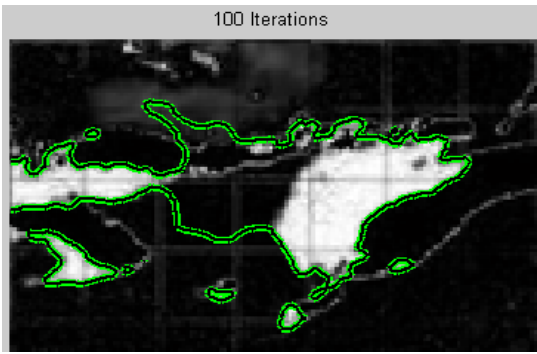


Figure 3. Result of segmentation with 100 iteration

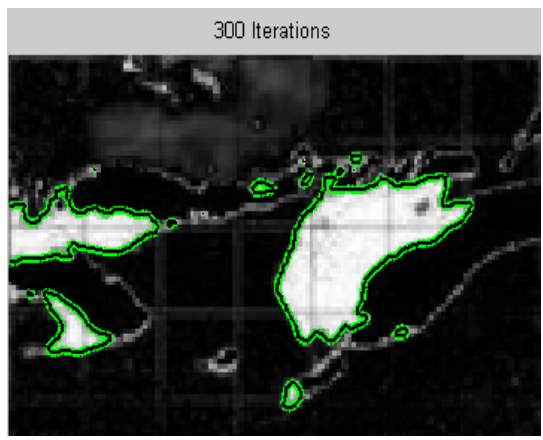

Figure 4. Result of segmentation with 300 iteration

The results shown in Figure 2 shows the separation boundary between the regions contained chlorophyll and the mainland use the edge indicator function. Figures 3 and 4 indicate that the approach only detects areas that contain chlorophyll.

\section{CONCLUSION}

We have presented active contour model based edge indicator for image satellite. This method can detect regions that contain chlorophyll effectiveness, quickly and accurately. With the method can be found quickly in areas that contain chlorophyll satellite image. Areas that contain chlorophyll can directly located by using this method. Therefore this method is very precise in segmenting regions that contain chlorophyll on satellite images.

\section{REFERENCES}

[1.] Ballangan Cherry., Adipranata Rudy., Nugroho William ., Image Segmentation Using Active Contour (Snake).,Seminar Nasional Sistem dan Informatika 2006; Bali, November 17, 2006 SNSI06-047.

[2.] Besbes Olfa ., Belhadj Ziad ., Boujemaa Nozha., Adaptive Satellite Images Segmentation by Level Set., Institut National De Recherche En Informatique Et En Automatique.,2006.

[3.] Ferreira, Monique Sacardo, Maria de Lourdes Bueno Trindade Galo. Use of World View-2 Imagery in Detection of Chlorophyll A. The Digital Globe 8-Band Research Challenge.,2011.

[4.] Lankton, Shawn, Allen Tannenbaum. Localizing Region-Based Active Contours. IEEE Transactions on Image Processing, vol. 17, no. 11, November 2008.

[5.] Lie J., Lysaker M., Tai X.C., A binary level set model and some application to Mumford-Shah image segmentation, IEEE Transaction on Image Processing 15(2006)1171-1181.

[6.] Nakhjavanlo B. Bagheri, Ellis T. J., Raoofi P., Dehmeshki J., Medical Image Segmentation Using Deformable Models and Local Fitting Binary.,world academy of science, Engineering and Technology.,2011

[7.] Rajeswari M.., Gurumurthy K.S.., Reddy L. Pratap., Omkar S.N., Senthilnath J., Automatic Road Extraction based on Level
Set,Normalized Cuts and Mean Shift Methods., IJCSI International Journal of Computer Science Issues, Vol. 8, Issue 3, No. 2, May 2011.,ISSN (Online): 1694-0814.

[8.] Reddy G.Raghotham., Ramudu K., Srinivas A., Rao R.Rameshwar., Fast Level Set Evolution of Region Based Segmentation of Satellite and Medical Imagery on Noisy Images., International Journal of Applied Physics and Mathematics, Vol. 1, No. 1, July 2011.

[9.] Singh Abhishek., Jaikumar Padmini., Mitra Suman K., Segmentation of Remotely Sensed Images Using Resampling Based Bayesian Learning., Journal of Pattern Recognition Research 1 (2010) 119-130.,2010

[10.] Sundaramoorthi, Ganesh, Anthony Yezzi, Andrea Menucci. Sobolev Active Contours. International Journal of Computer Vision 73(3).,2007

[11.] Wang, Li, Chunming Li, Quansen Sun, Deshen Xia , Chiu-Yen Kao. 2009. Active contours driven by local and global intensity fitting energy with application to brain MR image segmentation. Computerized Medical Imaging and Graphics 33 .,2009. 